\title{
Can High-Risk Fungicides be Used in Mixtures Without Selecting for Fungicide Resistance?
}

\author{
Alexey Mikaberidze, Bruce A. McDonald, and Sebastian Bonhoeffer
}

First, second, and third authors: Institute of Integrative Biology, ETH Zurich.

Accepted for publication 4 September 2013.

\begin{abstract}
Mikaberidze, A., McDonald, B. A., and Bonhoeffer, S. 2014. Can highrisk fungicides be used in mixtures without selecting for fungicide resistance? Phytopathology 104:324-331.

Fungicide mixtures produced by the agrochemical industry often contain low-risk fungicides, to which fungal pathogens are fully sensitive, together with high-risk fungicides known to be prone to fungicide resis-

of competition between the sensitive and resistant pathogen strains and to assess the usefulness of a mixture. If fitness costs are absent, then the use of the high-risk fungicide in a mixture selects for resistance and the fungicide eventually becomes nonfunctional. If there is a cost of resistance, then an optimal ratio of fungicides in the mixture can be found, at which selection for resistance is expected to vanish and the level of disease control can be optimized.
\end{abstract} tance. Can these mixtures provide adequate disease control while minimizing the risk for the development of resistance? We present a population dynamics model to address this question. We found that the fitness cost of resistance is a crucial parameter to determine the outcome
Additional keywords: epidemiology, host-pathogen interaction, mathematical model, plant disease.
Fungicide resistance is a prime example of adaptation of a population to an environmental change, also known as evolutionary rescue $(6,23)$. While global climate change is expected to result in a loss of biodiversity in natural ecosystems, evolutionary rescue is seen as a mechanism that may mitigate this loss. In the context of crop protection the point of view is quite the opposite: reducing adaptation of crop pathogens to chemical disease control would help stabilize food production. Better understanding of the adaptive process may help slow or prevent it. This requires a detailed quantitative understanding of the dynamics of infection and the factors driving the emergence and development of fungicide resistance (54). Despite the global importance and urgency of fungicide resistance, this problem has received relatively little theoretical consideration ([20,22,36,41,49,50] and [54] for a comprehensive review) as compared, for example, to antibiotic resistance $(4,10,33,39)$. In recent years, agrochemical companies have begun marketing mixtures that contain fungicides with a low risk of developing resistance with fungicides that have a high risk of developing resistance. In extreme cases the highrisk fungicide is no longer effective against some common pathogens because resistance has become widespread. For example, a large proportion of the European population of the important wheat pathogen Mycosphaerella graminicola (recently renamed Zymoseptoria tritici) $(38,40)$ is resistant to strobilurin fungicides (53).

A number of previous modeling studies addressed the effect of fungicide mixtures on selection for fungicide resistance $(21,22$, 26,27,48,51). Different studies used different definitions of "independent action" (also called "additivity" or "zero inter-

Corresponding author: A. Mikaberidze

E-mail address: alexey.mikaberidze@env.ethz.ch

* The $\boldsymbol{e}$-Xtra logo stands for "electronic extra" and indicates that the online version contains a supplemental appendix.

http://dx.doi.org/10.1094/PHYTO-07-13-0204-R

(C) 2014 The American Phytopathological Society action" in the literature) of fungicides in the mixtures (47) and reported somewhat different conclusions. One study (47) critically reviewed the outcomes of these earlier studies and attempted to clarify the consequences of using different definitions of independent action. Some studies found that alternations are preferable to mixtures (27), while others found that mixtures are preferable to alternations (51). A more recent study (21) addressed this question using a detailed population dynamics model and found that in all scenarios considered, mixtures provided the longest effective life of fungicides compared with alternations or concurrent use (when each field receives a single fungicide, but the fungicides applied differ between the fields). This study used the Bliss definition of independent action of the two fungicides (9) (also called Abbot's formula in the fungicide literature [1]).

We addressed the question of whether mixtures of low-risk and high-risk fungicides can provide adequate disease control while minimizing further selection for resistance using a simple population dynamics model of host-pathogen interaction based on a system of ordinary differential equations. We found that the fitness cost associated with resistance mutations is a crucial parameter, which governs the outcome of the competition between the sensitive and resistant pathogen strains.

A single point mutation associated with fungicide resistance sometimes makes the pathogen completely insensitive to a fungicide, as is the case for the G143A mutation giving resistance to strobilurin fungicides in many fungal pathogens $(14,16)$. In many other cases the resistance is partial, for example, resistance of $Z$. tritici and other fungi to azole fungicides $(12,37,57)$. Therefore, we considered varying degrees of resistance in our model.

In contrast to our study, resistance in Hobbelen et al. (22) was assumed to bear no fitness costs for the pathogen. It was found that in the absence of fitness costs the use of fungicide mixtures delays the development of resistance (22). This conclusion is in agreement with our results. Here we focus on finding conditions under which the selection for the resistant pathogen strain is prevented by using fungicide mixtures. 


\section{THEORY AND APPROACHES}

We use a deterministic mathematical model of susceptibleinfected dynamics (Fig. 1).

$$
\begin{gathered}
\frac{d H}{d t}=r_{H}\left(K-H-I_{\mathrm{s}}-I_{\mathrm{r}}\right)-b\left(\left[1-\varepsilon_{\mathrm{s}}\left(C, r_{\mathrm{B}}\right)\right] I_{\mathrm{s}}+\right. \\
\left.\left[1-\varepsilon_{\mathrm{r}}\left(C, r_{\mathrm{B}}\right)\right]\left(1-\rho_{\mathrm{r}}\right) I_{\mathrm{r}}\right) H \\
\frac{d I_{\mathrm{s}}}{d t}=b\left[1-\varepsilon_{\mathrm{s}}\left(C, r_{\mathrm{B}}\right)\right] H I_{\mathrm{s}}-\mu I_{\mathrm{s}} \\
\frac{d I_{\mathrm{r}}}{d t}=b\left[1-\varepsilon_{\mathrm{r}}\left(C, r_{\mathrm{B}}\right)\right]\left(1-\rho_{\mathrm{r}}\right) H I_{\mathrm{r}}-\mu I_{\mathrm{r}}
\end{gathered}
$$

The model has three compartments: healthy hosts $H$, hosts infected by a sensitive pathogen strain $I_{\mathrm{s}}$, hosts infected by a resistant pathogen strain $I_{\mathrm{r}}$; and is similar to the models described in Hall et al. (20) and van den Bosch and Gilligan (54). The subscript " $s$ " stands for the sensitive strain and the subscript " $r$ " stands for the resistant strain. The quantities $H, I_{\mathrm{s}}$, and $I_{\mathrm{r}}$, represent the total amount of the corresponding host tissue within one field, which could be leaves, stems, or grain tissue, depending on the specific host-pathogen interaction. Healthy hosts $H$ grow with the rate $r_{H}$. Their growth is limited by the "carrying capacity" $K$, which may imply limited space or nutrients. Furthermore, healthy hosts may be infected by the sensitive pathogen strain and transformed into infected hosts in the compartment $I_{\mathrm{S}}$ with the transmission rate $b$. This is a compound parameter given by the product of the sporulation rate of the infected tissue and the probability that a spore causes new infection. Healthy hosts may also be infected by the resistant pathogen strain and transformed into infected hosts in the compartment $I_{\mathrm{r}}$. In this case, resistant mutants suffer a fitness cost $\rho_{\mathrm{r}}$ which affects their transmission rate such that it becomes equal to $b\left(1-\rho_{\mathrm{r}}\right)$. The corresponding terms in equations 1 to 3 are proportional to the amount of the available healthy tissue $H$ and to the amount of the infected tissue $I_{\mathrm{s}}$ or $I_{\mathrm{r}}$. Infected host tissue loses its infectivity at a rate $\mu$, where $\mu^{-1}$ is the characteristic infectious period.

Since our description is deterministic we do not take into account the emergence of new resistance mutations but assume that the resistant pathogen strain is already present in the population. Therefore, when "selection for resistance" is discussed below, we refer to the process of winning the competition by this existing resistant strain due to its higher fitness with respect to the sensitive strain in the presence of fungicide treatment. Emergence of new resistance mutations is a different problem, which goes beyond the scope of our study and requires stochastic simulation methods. We do not consider the possibility of double resistance in the model, but by preventing selection for single resistance as described here, one would also diminish the probability of the emergence of double resistance for both sexually and asexually reproducing pathogens (Supplemental Appendix A.7).

We consider two fungicides, A and B. Fungicide A is the highrisk fungicide, to which the resistant pathogen strain exhibits a variable degree of resistance. However, the sensitive strain is fully sensitive to fungicide A. Fungicide B is the low-risk fungicide, i.e., both pathogen strains are fully sensitive to it. We compare the effects of fungicide A applied alone, fungicide B applied alone, and the effect of their mixture in different proportions.

We assume that the fungicides will decrease the pathogen transmission rate $b$ (see the expression in square brackets in equations 2 and 3). For example, application of a fungicide could result in production of spores that are deficient in essential metabolic products such as ergosterol or $\beta$-tubulin. Consequently, these spores would likely have a lower success rate in causing new infections. Spores of sensitive strains of $Z$. tritici produced shorter germ tubes when exposed to azoles (32). Spores that produce shorter germ tubes are less likely to find and penetrate stomata, hence are less likely to give rise to new infections. Protectant activity of fungicides will also reduce the transmission rate $b$ $(45,56)$. These studies $(45,56)$ also reported that fungicide application leads to a reduction in the number of spores produced. This outcome can be attributed to the fungicide decreasing the sporulation rate and thus affecting $b$ or decreasing the infectious period and thus affecting $\mu$, or both of these effects. More detailed measurements are often needed to distinguish between these different effects.

When only one fungicide is applied, the reduction of the transmission rate is described by

$$
\varepsilon_{\mathrm{A}}\left(C_{\mathrm{A}}\right)=k_{\mathrm{kA}} \frac{C_{\mathrm{A}}}{C_{\mathrm{A}}+C_{50 \mathrm{~A}}}
$$

for the fungicide A, and by

$$
\varepsilon_{\mathrm{B}}\left(C_{\mathrm{B}}\right)=k_{\mathrm{kB}} \frac{C_{\mathrm{B}}}{C_{\mathrm{B}}+C_{50 \mathrm{~B}}}
$$

for the fungicide $B$. These functions grow with the fungicide doses $C_{\mathrm{A}}$ and $C_{\mathrm{B}}$ and saturate to values $k_{\mathrm{kA}}$ and $k_{\mathrm{kB}}$, respectively, which are the maximum reductions in the transmission rate (or efficacies). This functional form was used before in the fungicide resistance literature $(18,20)$. We also performed the analysis using the exponential fungicide action more common in plant pathology

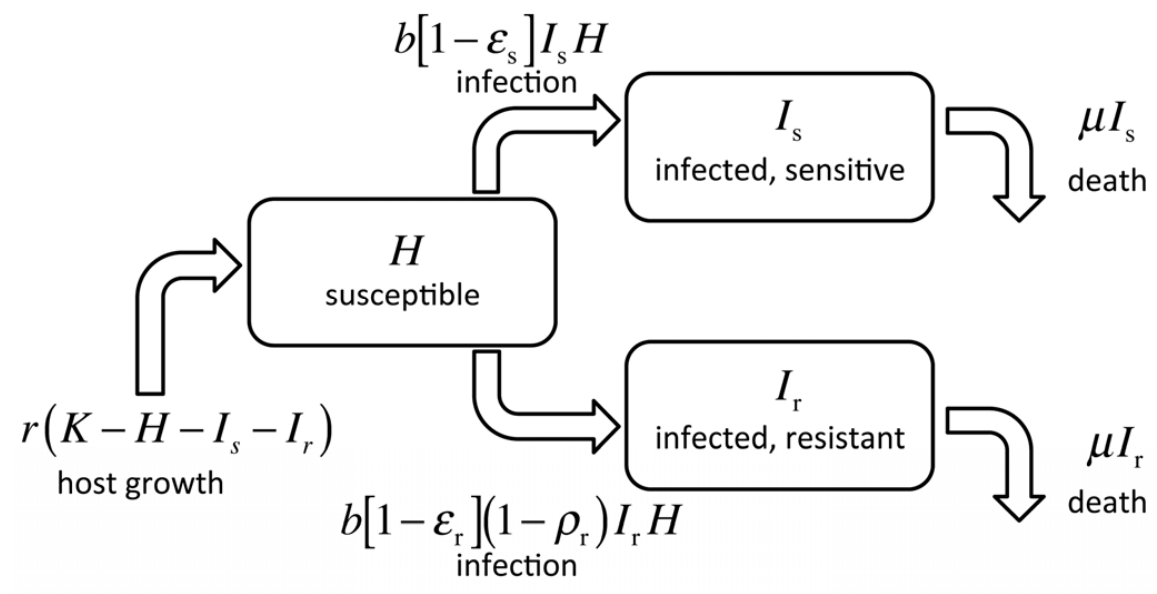

Fig. 1. Scheme of the model in equations 1 to 3 . 
and obtained qualitatively similar results. The reason for choosing the function in equation 5 was that it made it possible to obtain all the results analytically. The parameters $C_{50 \mathrm{~A}}$ and $C_{50 \mathrm{~B}}$ represent the fungicide dose at which half of the maximum effect is achieved. These parameters can always be made equal by rescaling the concentration axis for one of the fungicides. Hence, we set $C_{50 \mathrm{~A}}=C_{50 \mathrm{~B}}=C_{50}$.

We next determine the effect of a mixture of two fungicides according to Loewe's definition of additivity (or non-interaction) (7) (an equivalent graphical procedure is known as the Wadley method in the fungicide literature [34]). It is based on the notion that a compound cannot interact pharmacologically with itself. A sham mixture of a compound A with itself can be created and its effect used as a reference point for assessing whether the components of a real mixture interact pharmacologically. When the two compounds A and B have the same effect as the sham mixture of the compound A with itself, they are said to have no interaction (or an additive interaction). In this case, the isobologram equation

$$
C_{\mathrm{A}} / C_{\mathrm{Ai}}+C_{\mathrm{B}} / C_{\mathrm{Bi}}=1
$$

holds (see section VA of Berenbaum [7] for the derivation). Here, $C_{\mathrm{A}}$ and $C_{\mathrm{B}}$ are the doses of the compounds $\mathrm{A}$ and $\mathrm{B}$, respectively, when applied in the mixture; $C_{\mathrm{Ai}}$ is the isoeffective dose of the compound $\mathrm{A}$, that is the dose at which compound $\mathrm{A}$ alone has the same effect as the mixture; and $C_{\mathrm{Bi}}$ is the isoeffective dose of the compound $\mathrm{B}$. If the mixture of $\mathrm{A}$ and $\mathrm{B}$ has a larger effect than the zero-interactive sham mixture, then $C_{\mathrm{A}} / C_{\mathrm{Ai}}+C_{\mathrm{B}} / C_{\mathrm{Bi}}<1$ and the two compounds are said to interact synergistically. On the other hand, when the mixture of $\mathrm{A}$ and $\mathrm{B}$ has a smaller effect than the zero-interactive sham mixture, $C_{\mathrm{A}} / C_{\mathrm{Ai}}+C_{\mathrm{B}} / C_{\mathrm{Bi}}>1$, the two compounds interact antagonistically.

Using the dose-response dependencies of each fungicide when applied alone, equations 5 and 6, we derive the dose-response function for the combined effect of the two fungicides on the sensitive pathogen strain in the case of no pharmacological interaction (see section VIB of Berenbaum [7] for the derivation):

$$
\varepsilon_{\mathrm{s}}\left(C_{\mathrm{A}}, C_{\mathrm{B}}\right)=\frac{k_{\mathrm{kA}} C_{\mathrm{A}}+k_{\mathrm{kB}} C_{\mathrm{B}}}{C_{\mathrm{A}}+C_{\mathrm{B}}+C_{50}}
$$

Similarly, we determine the combined effect of the two fungicides on the resistant pathogen strain without pharmacological interaction:

$$
\varepsilon_{\mathrm{r}}\left(C_{\mathrm{A}}, C_{\mathrm{B}}\right)=\frac{k_{\mathrm{kA}} \alpha C_{\mathrm{A}}+k_{\mathrm{kB}} C_{\mathrm{B}}}{\alpha C_{\mathrm{A}}+C_{\mathrm{B}}+C_{50}}
$$

where we introduced $\alpha$, the degree of sensitivity of the resistant strain to the fungicide A (the high-risk fungicide). At $\alpha=0$, the pathogen is fully resistant to fungicide $A$ and the effect of the mixture $\varepsilon_{\mathrm{r}}\left(C_{\mathrm{A}}, C_{\mathrm{B}}\right)$ in equation 8 does not depend on its dose $C_{\mathrm{A}}$, while at $\alpha=1$ the pathogen is fully sensitive to fungicide $\mathrm{A}$.

The expressions in equations 7 and 8 are only valid in the range of fungicide concentrations, over which isoeffective concentrations can be determined for both fungicides. Here, the isoeffective concentration is the concentration of a fungicide applied alone that has the same effect as the mixture. This requirement means that we are only able to consider the effect of the mixture at a sufficiently low total concentration: $C=C_{\mathrm{A}}+$ $C_{\mathrm{B}}<k_{\mathrm{kB}} C_{50} /\left(k_{\mathrm{kA}}-k_{\mathrm{kB}}\right) /\left(1-r_{\mathrm{B}}\right)$.

Next, we introduce deviations from the additive pharmacological interaction. There are several ways to do this, usually by adding an interaction term to the isobologram equation (17). We chose a specific form of the interaction term, which is proportional to the square root of the product of the concentrations of the two compounds (equation 28 in Greco and Bravo [17]). Assuming $k_{\mathrm{kA}}=k_{\mathrm{kB}}=k_{\mathrm{k}}$, this form allows for a simple analyti- cal expression for the effect of the combination on the sensitive strain

$$
\varepsilon_{\mathrm{s}}\left(C, r_{\mathrm{B}}\right)=k_{\mathrm{k}} \frac{C}{C+C_{50} / \gamma_{\mathrm{s}}}
$$

and on the resistant strain

$$
\varepsilon_{\mathrm{r}}\left(C, r_{\mathrm{B}}\right)=k_{\mathrm{k}} \frac{C}{C+C_{50} / \gamma_{\mathrm{r}}}
$$

Here $C=C_{\mathrm{A}}+C_{\mathrm{B}}$, where $C_{\mathrm{A}}$ is the dose of the fungicide $\mathrm{A}$ and $C_{\mathrm{B}}$ is the dose of the fungicide $\mathrm{B}, r_{\mathrm{B}}=C_{\mathrm{B}} / C$ is the proportion of the fungicide $\mathrm{B}$ in the mixture and

$$
\begin{gathered}
\gamma_{\mathrm{s}}=1+u \sqrt{r_{\mathrm{B}}\left(1-r_{\mathrm{B}}\right)} \\
\gamma_{\mathrm{r}}=\alpha\left(1-r_{\mathrm{B}}\right)+r_{\mathrm{B}}+u \sqrt{\alpha r_{\mathrm{B}}\left(1-r_{\mathrm{B}}\right)}
\end{gathered}
$$

are the parameters which modify $C_{50}$ due to pharmacological interaction and partial resistance. Equations 9 and 10 are obtained from equation 6 with an interaction term added and the doseresponse functions of each fungicide when applied alone, equations 4 and 5. The degree of pharmacological interaction is characterized by the parameter $u$. At $u=0$ the fungicides do not interact and equations 9 and 10 are the same as equations 7 and 8 . The case when $u>0$ represents synergy: the interaction term proportional to $u$ in equations 9 and 10 is positive and it reduces the value of $C_{50}$, meaning that the same effect can be achieved at a lower dose than at $u=0$. The case when $u<0$ corresponds to antagonism. Note, that the interaction term is proportional to $\sqrt{r_{\mathrm{B}}\left(1-r_{\mathrm{B}}\right)}$. This functional form guarantees that it vanishes, whenever only one of the compounds is used, i.e., $r_{\mathrm{B}}=0$ or $r_{\mathrm{B}}=1$.

In order to make clear the questions we ask and the assumptions we make, we consider the dynamics of the frequency of the resistant strain $p(t)=I_{\mathrm{r}}(t) /\left[I_{\mathrm{r}}(t)+I_{\mathrm{s}}(t)\right]$. The rate of its change is obtained from equations 1 to 3

$$
\frac{d p}{d t}=s(t) p(1-p)
$$

where

$$
s=b\left[\left(1-\varepsilon_{\mathrm{r}}\left(C, r_{\mathrm{B}}\right)\right)\left(1-\rho_{\mathrm{r}}\right)-\left(1-\varepsilon_{\mathrm{s}}\left(C, r_{\mathrm{B}}\right)\right)\right] H(t)
$$

is the selection coefficient (a similar expression was found in Gubbins and Gilligan [18]). Here $\varepsilon_{\mathrm{S}}\left(C, r_{\mathrm{B}}\right)$ and $\varepsilon_{\mathrm{r}}\left(C, r_{\mathrm{B}}\right)$ are given by equations 9 and 10. If $s>0$, then the resistant strain is favored by selection and will eventually dominate the pathogen population $(p \rightarrow 1$ as $t \rightarrow \infty)$. Alternatively, if $s<0$, then the sensitive strain is selected and will dominate the population $(p \rightarrow 0$ as $t \rightarrow \infty)$.

The focus of this paper is to investigate the parameter range over which $s<0$, i.e., the sensitive strain is favored by selection. Mathematically this corresponds to finding the range of stability of the equilibrium (fixed) point of the system equations 1 to 3 , corresponding to $H>0, I_{\mathrm{s}}>0, I_{\mathrm{r}}=0$. Our focus is mainly on the direction of selection. To address this point we do not need to assume that the host-pathogen equilibrium is reached. However, we explicitly assume that the host-pathogen equilibrium is reached during one season in the treatment benefits section, where we evaluate the benefit of fungicide treatment. The implications of this assumption are discussed at the beginning of the treatment benefits section. Furthermore, we assume that the fungicide dose is constant over time.

A careful examination of equation 14 reveals that the sign of the selection coefficient $s$, and therefore the direction of selection, is determined by the expression in square brackets, which can be 
either positive or negative depending on the values of $C, r_{\mathrm{B}}, \rho_{\mathrm{r}}$, and the shapes of the functions $\varepsilon_{\mathrm{s}, \mathrm{r}}$. The sign of the selection coefficient is unaffected by $b$ and $H(t)$ since both of them are nonnegative. Consequently, most of the results of this paper do not depend on a particular shape of $H(t)$ and hence are independent of a particular form of the growth term (except for those in the treatment benefits section). This means that the main conclusions of the paper remain valid for both perennial crops, where the amount of healthy host tissue steadily increases over many years, and for annual crops, where the healthy host tissue changes cyclically during each growing season.

We also neglect the spatial dependencies of the variables $H, I_{\mathrm{s}}$, and $I_{\mathrm{r}}$ and all other parameters. The latent phase of infection, which can be considerable for some pathogens, is also neglected. Since we neglect mutation, migration, and spatial heterogeneity, the resistant and sensitive pathogen strains cannot coexist in the long term. Only one of them eventually survives: the one with a higher basic reproductive number.

The basic reproductive number, $R_{0}$, is often used in epidemiology as a measure of transmission fitness of infectious pathogens (2). It is defined as the expected number of secondary infections resulting from a single infected individual introduced into a susceptible (healthy) population. At $R_{0}>1$ the infection can spread over the population, while at $R_{0}<1$ the epidemic dies out.

The equilibrium stability analysis of the model equations 1 to 3 (Supplemental Appendix A.1) shows that the relationship between the basic reproductive number of the sensitive strain $R_{0 \mathrm{~s}}=$ $b\left(1-\varepsilon_{\mathrm{S}}\left(C, r_{\mathrm{B}}\right)\right) K / \mu$ and the basic reproductive number of the resistant strain $R_{0 \mathrm{r}}=b\left(1-\varepsilon_{\mathrm{r}}\left(C, r_{\mathrm{B}}\right)\right)\left(1-\rho_{\mathrm{r}}\right) K / \mu$ determines the long-term outcome of the epidemic. The sensitive strain wins the competition and dominates the pathogen population if $R_{0 \mathrm{~s}}>1$, such that it can survive in the absence of the resistant strain, and $R_{0 \mathrm{~s}}>R_{0 \mathrm{r}}$ (this is equivalent to $s<0$ ), such that it has a selective advantage over the resistant strain. The latter inequality is equivalent to

$$
\varepsilon_{\mathrm{s}}\left(C, r_{\mathrm{B}}\right)<\rho_{\mathrm{r}}+\varepsilon_{\mathrm{r}}\left(C, r_{\mathrm{B}}\right)\left(1-\rho_{\mathrm{r}}\right)
$$

Similarly, the resistant strain wins the competition and dominates the population if $R_{0 \mathrm{r}}>1$ and $R_{0 \mathrm{r}}>R_{0 \mathrm{~s}}$ (this is equivalent to $s>0$ ).

We determined the range of the fungicide doses $C$ and fitness costs $\rho_{\mathrm{r}}$, according to the inequality (15) analytically when (i) the high-risk fungicide has a higher efficacy than the low-risk fungicide $\left(k_{\mathrm{kA}}>k_{\mathrm{kB}}\right)$, but pharmacological interaction is absent $(u=0)$; and (ii) the two fungicides have the same efficacy $\left(k_{\mathrm{kA}}=k_{\mathrm{kB}}=k_{\mathrm{k}}\right)$, but may interact pharmacologically $(u \neq 0)$. In case (i) the criterium (14) assumes the form

$$
\frac{C}{C+C_{50}}<\rho_{\mathrm{r}} / k_{\mathrm{km}}+k_{\mathrm{kB}} / k_{\mathrm{km}} \frac{C}{C+C_{50} / \gamma_{\mathrm{r}}}\left(1-\rho_{\mathrm{r}}\right)
$$

where $k_{\mathrm{km}}=k_{\mathrm{kA}}\left(1-r_{\mathrm{B}}\right)+k_{\mathrm{kB}} r_{\mathrm{B}}$, while in case (ii) the criterium (14) reads

$$
\frac{C}{C+C_{50} / \gamma_{\mathrm{s}}}<\rho_{\mathrm{r}} / k_{\mathrm{k}}+\frac{C}{C+C_{50} / \gamma_{\mathrm{r}}}\left(1-\rho_{\mathrm{r}}\right)
$$

To keep the presentation concise, below we present the results corresponding to case (ii), i.e., solve the inequality (17). However, we verified that all the conclusions remain the same in case (i). In a more general case, when $k_{\mathrm{kA}}>k_{\mathrm{kB}}$ and $u \neq 0$ the parameter ranges satisfying the inequality (15) can only be determined numerically.

We assume here that both the cost of resistance and fungicidal activity decrease the transmission rate $b$. However, we performed the same analysis when the effect of the resistance cost and the fungicide enter the model in other ways and obtained qualitatively similar results (Supplemental Appendix A.5).
The simplicity of the model allows us to obtain all of the results analytically. We determined explicit mathematical relationships between the quantities of interest, which enabled us to study the effects over the whole range of parameters.

\section{RESULTS}

We first investigate the parameter ranges over which resistant or sensitive strains dominate the pathogen population for the case of fungicides A and B applied individually and in a mixture. Then, we consider the optimal proportion of fungicides to include in a mixture and the benefit of fungicide treatment. Finally, we take into account possible pharmacological interactions between fungicides and consider the effect of partial resistance.

Selection for resistance. The ranges of fungicide dose and cost of resistance at which the sensitive (white) or resistant (gray) pathogen strain is favored by selection are shown in Figure 2. In all scenarios competitive exclusion is observed: one of the strains takes over the whole pathogen population and the other one is eliminated. If a low-risk fungicide is applied alone, the sensitive strain has a selective advantage across the whole parameter range in Figure 2A. When only a high-risk fungicide is applied (Fig. 2B), the resistance dominates if the fitness cost is lower than the maximum effect of the fungicide $\rho_{\mathrm{r}}<k_{\mathrm{k}}$ and at a fungicide dose higher than a threshold value which increases with the fitness cost (solid curve in Fig. 2B). If the fitness cost exceeds $k_{\mathrm{k}}$ (dotted line in Fig. 2B), then the sensitive strain dominates at any fungicide dose. Figure $2 \mathrm{C}$ shows the outcome when the two fungicides are mixed at equal concentrations. Here the fitness cost at which the sensitive strain dominates is reduced (vertical dotted line is shifted to the left).

As expected, without a fitness cost $\left(\rho_{\mathrm{r}}=0\right)$ the resistant strain becomes favored by selection and will eventually dominate the population whenever the high-risk fungicide is applied, alone or in combination, with the low-risk fungicide (Fig. 2B and C).

Optimal proportion of fungicides in a mixture. It is highly desirable to keep existing fungicides effective for as long as possible. From this point of view, an optimal mixture contains the

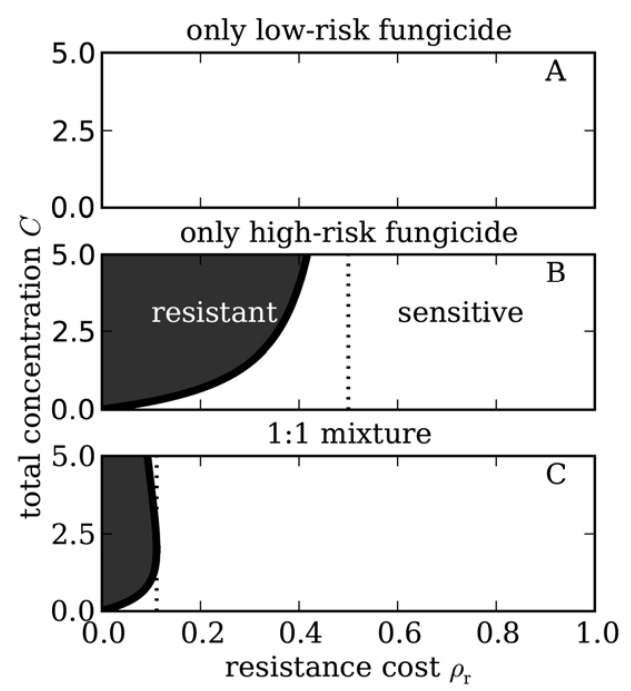

Fig. 2. Outcomes of the competition between the sensitive and resistant pathogen strains depending on the fitness cost of resistance $\rho_{\mathrm{r}}$ and the fungicide dose $C$ when treated with $\mathbf{A}$, a single fungicide $\mathrm{B}$ at $\left[C_{\mathrm{B}}=C\right], \mathbf{B}$, a single fungicide $\mathrm{A}\left[C_{\mathrm{A}}=C\right]$, and $\mathbf{C}$, the combination of fungicides $\mathrm{A}$ and $\mathrm{B}$ $\left[C_{\mathrm{A}}=C_{\mathrm{B}}=C / 2\right]$. The range of the total fungicide dose $C$ and the fitness cost of resistance $\rho_{\mathrm{r}}$, in which the resistant strain is favored is shown in dark gray. The range where selection favors the sensitive strain is shown in white. Fungicides are assumed to have zero interaction $(u=0)$ and the resistant strain is assumed to be fully protected from fungicide $\mathrm{A}(\alpha=0)$. The fungicide dose-response parameters are $k_{\mathrm{k}}=0.5, C_{50}=1$. 
largest proportion of the high-risk fungicide, at which (i) the resistant pathogen strain is not selected and (ii) an adequate level of disease control is achieved. In order to fulfill both of these objectives, the fitness cost of resistance needs to be larger than a threshold value $\rho_{\mathrm{r}}>\rho_{\mathrm{rb}}$. The threshold $\rho_{\mathrm{rb}}$ is shown by the dotted vertical line in Figure 2C.

The threshold $\rho_{\mathrm{rb}}$ depends on the proportion of fungicides in the mixture. Adding more of the low-risk fungicide, while keeping the same total dose $C$, reduces the threshold. This diminishes the range of the values for fitness cost over which the resistant strain dominates. On the other hand, adding less of the low-risk fungicide, while again keeping $C$ the same, increases the threshold, which increases the parameter range over which the resistant strain is favored.

Therefore, at a given fitness cost $\rho_{\mathrm{r}}$, one can adjust the fungicide ratio $r_{\mathrm{B}}$ such that $\rho_{\mathrm{r}}>\rho_{\mathrm{rb}}$. This is shown in Figure 3: the curve shows the critical proportion of the low-risk fungicide $r_{\mathrm{Bc}}$, above which no selection for resistance occurs at any total fungicide dose $C$. One can see from Figure 3 that if the resistance cost is absent $\left(\rho_{\mathrm{r}}=0\right)$, then the high-risk fungicide should not be added at all if one wants to prevent selection for resistance. At larger fitness costs, the value of $r_{\mathrm{Bc}}$ decreases, giving the possibility to use a larger proportion of the high-risk fungicide without selecting for resistance.

Finding an optimum proportion of fungicides requires knowledge of both the fitness cost $\rho_{\mathrm{r}}$ and the maximum effect of the fungicide $k_{\mathrm{k}}$. However, if the cost of resistance and fungicides affect the infectious period of the pathogen $\mu^{-1}$ and not the transmission rate $b$ as we assumed above, then a simpler expression for the critical proportion of fungicides in the mixture is obtained, which depends only on the ratio between the fitness cost and the maximum fungicide effect $\rho_{\mathrm{r}} / k_{k}$. In this case, if the fitness cost is at least $5 \%$ of the maximum fungicide effect, then we predict that up to about $20 \%$ of the high-risk fungicide can be used in a mixture without selecting for resistance. An example of the cost of fungicide resistance manifesting as a reduction in infectious period was in metalaxyl-resistant isolates of Phytophthora infestans (28). In this experiment, the infectious period of the resistant isolates was reduced, on average, by $25 \%$ compared with the susceptible isolates (28).

So far we have shown how choosing an optimal proportion of fungicides in the mixture prevents selection for resistance. Now, we will consider in more detail how to achieve an adequate level of disease control.

Treatment benefit. The yield of cereal crops is usually assumed to be proportional to the healthy green leaf area, which

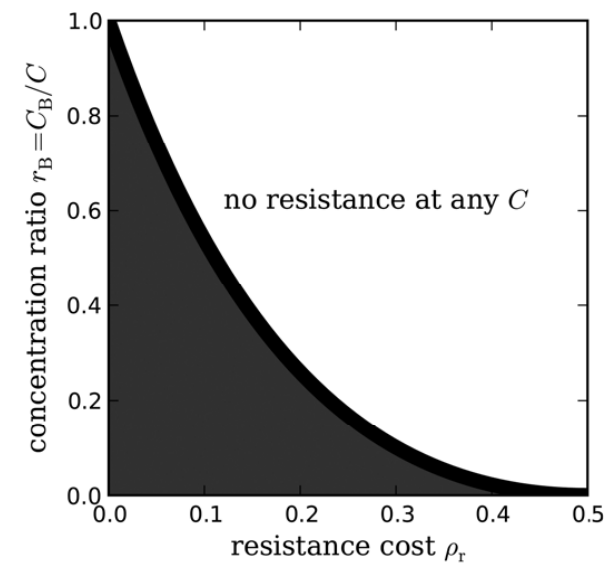

Fig. 3. The critical proportion $r_{\mathrm{Bc}}$ of fungicide $\mathrm{B}$ (low-risk fungicide) in the mixture, above which there is no selection for the resistant strain at any total fungicide dose $C$, plotted (black curve) as a function of the resistance cost $\rho_{\mathrm{r}}$, assuming no pharmacological interaction $(u=0)$, full resistance $(\alpha=0)$, and the maximum fungicide effect $k_{\mathrm{k}}=0.5$. corresponds in our model to the amount of healthy hosts $H(t)$. Accordingly, we quantify the benefit of the fungicide treatment, $B(t)$, as the ratio between the amount of healthy hosts $H(t)$ when both the disease and treatment are present and its value $H_{\text {nd }}(t)$ in the absence of disease: $B(t)=H(t) / H_{\mathrm{nd}}(t)$. Hence, $B(t)=1$ corresponds to a perfect treatment, which eradicates the disease completely and the treatment benefit of zero corresponds to a situation where all healthy hosts are infected by disease. In order to obtain analytical expressions for the treatment benefit $B(t)$, we consider one growing season and assume that the host-pathogen equilibrium is reached during the season.

The treatment benefit at equilibrium is shown in Figure 4 as a function of the fitness cost and the fungicide dose (see Supplemental Appendix A.3 for equations). When a low-risk fungicide is applied alone (Fig. 4A), the sensitive strain is favored by selection over the whole range of parameters. Therefore, the treatment benefit increases monotonically with the fungicide dose and is not affected by the cost of resistance. In contrast, when a high-risk fungicide is applied alone (Fig. 4B), a region at low fitness cost appears (Fig. 4B, left of the solid curve), where the resistant strain is favored. Here, the treatment benefit does not depend on the fungicide dose, but increases with the cost of resistance. Hence, if the fitness cost is too low to stop selection for resistance, then the fungicide treatment will fail.

In the case of a mixture of a high-risk and a low-risk fungicide, the parameter range over which the resistant strain is favored becomes smaller (Fig. 4C, left of the solid curve). In this range the treatment benefit increases with the cost of resistance, since larger costs reduce the impact of disease per se. Also, the treatment benefit increases with the total fungicide dose in this range, because the low-risk fungicide works against the resistant strain.

As we showed earlier, in the presence of a substantial fitness cost, one can avoid selection for resistance by adjusting the proportion of the two fungicides in the mixture. In this case, the total fungicide dose should be chosen such that the treatment benefit reaches a high enough value and an adequate level of disease control is achieved.

We estimated earlier that up to about $20 \%$ of the high-risk fungicide can be used in a mixture without selecting for resistance if the fitness cost is at least $5 \%$ of the maximum fungicide effect

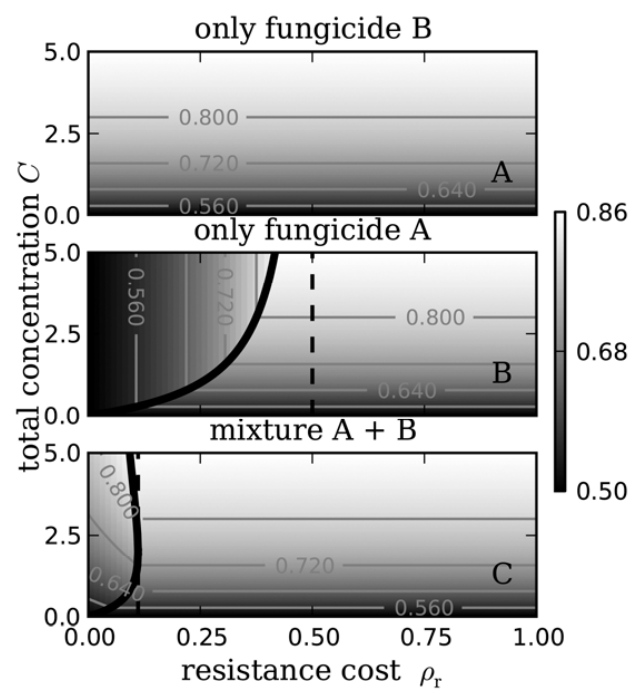

Fig. 4. Treatment benefit as a function of fungicide dose $C$ and fitness cost of resistance $\rho_{\mathrm{r}}$. A, Treatment with fungicide B. B, Treatment with fungicide A. $\mathbf{C}$, Treatment with a mixture of $\mathrm{A}$ and $\mathrm{B}$ at equal concentrations $\left(r_{\mathrm{B}}=1 / 2\right)$. Solid and dashed curves in panels $\mathrm{B}$ and $\mathrm{C}$ are the same as in Figure 2. Fungicides are assumed to have zero interaction $(u=0)$ and the resistant strain is assumed to be fully protected from fungicide $\mathrm{A}(\alpha=0)$. The fungicide doseresponse parameters are $k_{\mathrm{k}}=0.5, C_{50}=1$, the basic reproductive number of the sensitive strain without fungicide treatment $R_{0 \mathrm{~s}}(C=0)=b K / \mu=2$. 
on the infectious period $\mu^{-1}$. But how much extra control does one obtain by adding the high-risk fungicide to the mixture? We estimate that adding $20 \%$ of the high-risk fungicide to the mixture increases the treatment benefit by about $12 \%$ at $R_{0 \mathrm{~s}}(C=0)=$ $b K / \mu=4$ and by about $9 \%$ at $R_{0 \mathrm{~s}}(C=0)=2$. In the case when the high-risk fungicide has a larger maximum effect, i.e., $k_{\mathrm{kA}}>k_{\mathrm{kB}}$, the benefit of adding it to the mixture will increase. However, the largest proportion of the high risk that can be added without selecting for resistance will decrease.

The effect of pharmacological interaction between fungicides. Synergistic interactions between fungicides make their combined effect greater than expected with additive interactions. The sensitive pathogen strain is suppressed more by a synergistic mixture, while the resistant strain is not affected by the interaction (in case of full resistance $\alpha=0$ ). This increases the range of fitness costs over which resistance has a selective advantage (the dashed line in Figure 2C shifts to the right). Consequently, the critical proportion of the low-risk fungicide in the mixture $r_{\mathrm{Bc}}$, above which the resistant mutants are eliminated increases (Fig. $5 \mathrm{~A}$, dotted curve). In contrast, an antagonistic mixture suppresses the sensitive strain less effectively than either fungicide used alone. In this case the range of fitness costs over which resistance dominates becomes smaller and the ratio $r_{\mathrm{Bc}}$ decreases (Fig. 5A, dashed curve). Hence, reduced resistance evolution is achieved, but at the expense of reduced disease control. This result is in agreement with studies on drug interactions in the context of antibiotic resistance, where antagonistic drug combinations were found to select against resistant bacterial strains (11).

The effect of partial fungicide resistance. Consider the situation when the resistant pathogen strain is not fully protected from the high-risk fungicide, but exhibits a partial resistance $(0<\alpha<1)$. In this case, the fungicide mixture is more effective in suppressing the resistant strain than in the case of full resistance $(\alpha=0)$ considered above. Therefore, one needs less of the low-risk fungicide in the mixture to reach the conditions where resistance is eliminated by selection: the critical proportion of the low-risk fungicide in the mixture decreases with the degree of sensitivity $\alpha$ in Figure 5B. In Figure 5A the dependency of the critical ratio of the fungicide $\mathrm{B}$ in the mixture for partial resistance (light gray curve) lies below the one at perfect resistance and reaches zero at a much smaller value. Thus, knowledge of the degree of resistance is crucial for determining an appropriate proportion of fungicides in the mixture.

\section{DISCUSSION}

The three main outcomes of our study are as follows: (i) if fungicide resistance comes without a fitness cost, application of fungicides prone to resistance (high-risk fungicides) in a mixture with fungicides still free from resistance (low-risk fungicides) will select for resistance; (ii) if sufficiently high costs are found, then an optimal proportion of the high-risk fungicide in a mixture with the low-risk fungicide exists that does not select for resistance; and (iii) this mixture can potentially be used for preventing de novo emergence of fungicide resistance, in which case the relevant fitness cost is the "inherent" cost of fungicide resistance before compensatory evolution occurs (described below).

In the absence of fitness costs, application of a mixture of highrisk and low-risk fungicides will select for resistance. Consequently, the resistant strain will eventually dominate the pathogen population and the sensitive strain will be eliminated. Because of this, the high-risk fungicide will not affect the amount of disease and only the low-risk fungicide component of the mixture will be acting against disease. Hence, the high-risk fungicide becomes nonfunctional in the mixture and using the low-risk fungicide alone would have the same effect at a lower financial and environmental cost.

In contrast, if sufficiently high costs are found, then high-risk fungicides can be used effectively for an extended period of time. According to our model, an optimal proportion of the high-risk fungicide in a mixture with the low-risk fungicide can be determined that contains as much as possible of the high-risk fungicide, but still does not select for resistance while providing adequate disease control (Table 1). If a mixture with the optimum proportion is applied, then the rise of the resistant strain is prevented for an unlimited time. Thus, the scheme in Table 1 provides a framework for using our knowledge about the evolutionary dynamics of plant pathogens and their interaction with fungicides to devise practical strategies for management of fungicide resistance.

TABLE 1. According to our model one can avoid selection for resistance while providing adequate disease control by choosing the fungicide ratio $r_{\mathrm{B}}$ and the total dose $C$ in the following way

\footnotetext{
1. Measure the pharmacological properties of both fungicides under field conditions to determine $k_{\mathrm{k}}$ and $C_{50}$.

2. Determine the degree of fungicide sensitivity $\alpha$ under field conditions.

3. Determine the degree of pharmacological interaction $u$ between fungicides $\mathrm{A}$ and $\mathrm{B}$ under field conditions.

4. Measure the fitness cost of resistance $\rho_{\mathrm{r}}$ under field conditions.

5. Choose the proportion of the fungicide $\mathrm{B}$ above the threshold: $r_{\mathrm{B}}>r_{\mathrm{Bc}}$, such that the resistance is not favored by selection at any total fungicide dose $C$.

6. Choose the total fungicide dose, which should be large enough to achieve an adequate level of disease control (Fig. 4C).
}
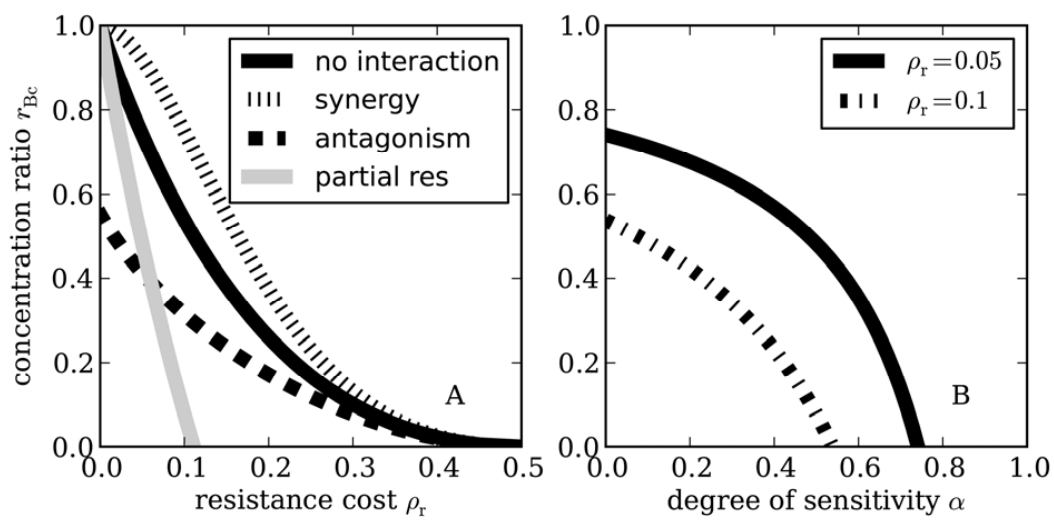

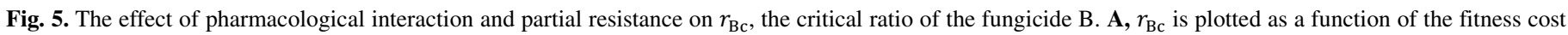

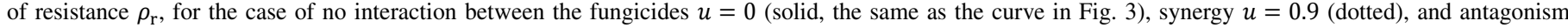

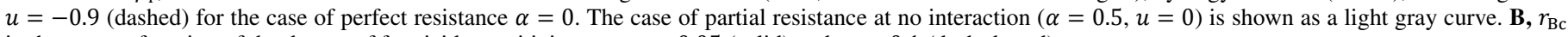
is shown as a function of the degree of fungicide sensitivity $\alpha$ at $\rho_{\mathrm{r}}=0.05$ (solid) and $\rho_{\mathrm{r}}=0.1$ (dash-dotted). 
In order to apply the scheme in Table 1, one needs to know dose-response parameters of the fungicides $k_{\mathrm{k}}$ and $C_{50}$, the degree of fungicide sensitivity $\alpha$ (or the resistance factor), the degree of pharmacological interaction $u$, and the fitness cost of resistance mutations. Fungicide dose-response curves are routinely determined empirically (for example $[35,42]$ ) and can be used to estimate the model parameters $k_{\mathrm{k}}$ and $C_{50}(22)$. The fungicide sensitivity is known to be lost completely in some cases (for example, most cases of QoI resistance), i.e., $\alpha=0$, while in other cases with partial resistance the degree of sensitivity (or the resistance factor) was measured (for example [32]). Pharmacological interaction between several different fungicides was also characterized empirically (Gisi [15] and the references therein). Also, the fitness costs of resistance were characterized empirically in many cases (described below). In the past these measurements were performed independently, but our study provides motivation to bring them together, since all these parameters need to be characterized for the same plant-pathogen-fungicide combination.

These measurements will allow one to predict the optimal proportion of the two fungicides in the mixture theoretically. This prediction needs to be tested using field experimentation, in which the amount of disease and the frequency of resistance would be measured as functions of time at different proportions of the high- and low-risk fungicides in the mixture. From these measurements the optimal proportion of the fungicides can be obtained empirically. It is this empirically determined optimal proportion of fungicides that can be used for practical guidance on management of fungicide resistance. Moreover, from the comparison of the optimal proportions obtained theoretically and empirically, one can evaluate the performance of the model and identify the aspects of the model that need improvement.

So far we considered the scenario where both the sensitive and the resistant pathogen strains increase from low numbers, i.e., resistant mutants pre-exist in the pathogen population. In this scenario the strain with higher fitness (or basic reproductive number) eventually outcompetes the other strain. This competition may occur over a time scale of several growing seasons so that there is enough time for compensatory mutations that diminish fitness costs of resistance to emerge. This needs to be taken into account when determining the optimal proportion of fungicides in the mixture. However, an alternative scenario is possible when resistant mutants emerge de novo through mutation or migration and, in order to survive, they need to invade the host population already infected by the sensitive strain. The threshold of invasion in this case depends on the "inherent" fitness cost of resistance mutations, i.e., their cost before the compensatory evolution occurs. In this case, one should measure the "inherent" cost of resistance mutations when performing step 4 in Table 1.

As discussed above, it is crucial to know fitness costs of resistance mutations in order to determine whether the fungicide mixture will select for resistance. We extensively searched the literature on fitness costs in different fungal pathogens of plants. A few studies inferred substantial fitness costs from field monitoring (for example [52] and references in [43]). But these findings could result from other factors, including immigration of sensitive isolates, selection for other traits linked to resistance mutations or genetic drift (43). Though relatively few carefully controlled experiments have been conducted, the majority indicate that fitness costs associated with fungicide resistance are either low (for example [8,31]) or absent (for example [13,44]). But in some cases fitness costs were found to be substantial (for example $[24,25,29,30,55])$ both in laboratory measurements and in field experiments. Although measurements of fitness costs of resistant mutants performed under laboratory conditions can be informative (for example [8]), they do not necessarily reflect the costs connected with resistant mutants selected in the field. This is because field mutants are likely to possess compensatory mutations improving pathogen fitness (43). Moreover, a labora- tory setting rarely reflects the balance of environmental and host conditions found throughout the pathogen life-cycle, since the field environment is much more complex.

However, the most relevant measure of pathogen fitness in the context of our study is the growth rate of the pathogen population at the very beginning of an epidemic (often denoted as $r$ ). It is directly related to the basic reproductive number $R_{0}$. To the best of our knowledge, the fitness costs of fungicide resistant strains were not measured with respect to $r$. In the studies cited above different components of fitness were measured that may or may not be related to $r$. Therefore, we identified a major gap in our knowledge of fitness costs. We hope this study will stimulate further experimental investigations to better characterize fitness costs and expect that substantial costs will be found in some cases.

Interactions of plants with fungal pathogens, fungicide action and, possibly, pharmacological interaction can depend on environmental conditions. This means that the outcomes of measurements necessary for applying the scheme in Table 1 may vary between seasons and geographical locations. Moreover, the outcomes may also be different in different host cultivars. Therefore, the optimal proportion of fungicides in the mixture may vary between seasons, geographical locations, and host cultivars. Thus, to provide general practical guidance on management of fungicide resistance, one needs to measure the optimal proportion of fungicides over many seasons, in different geographical locations and host cultivars. This difficulty is not a unique property of our study, but rather it is a general problem in the field of mathematical modeling of fungicide resistance and plant diseases. For example, it is also relevant for choosing appropriate fungicide dose rate (35).

While it was previously discussed (50) whether alternation of high-risk and low-risk fungicides might be a useful tactic for disease control in the presence of a fitness cost, we have shown that a mixture of these fungicides in an appropriate proportion can provide adequate disease control without selecting for resistance. Mixtures offer an advantage compared to alternation because there is no need to delay the application of the high-risk fungicide and the resistant strain does not rise to high frequencies, which lowers the risk of its further spread.

The strategy of combining chemical biocides in order to delay or prevent the development of resistance also appears in other contexts, including resistance of agricultural weeds to herbicides (5) and insect pests to insecticides (46). The fitness cost of resistance is also recognized as a crucial parameter for managing antibiotic resistance (3).

Development of mathematical models of fungicide resistance dynamics has been influenced by theoretical insights from animal and human epidemiology $(19,54)$. Similarly, we expect that lessons learned from modeling fungicide combinations may well apply to the problem of biocide resistance in the other contexts. In particular, one can investigate the idea of adjusting the proportion of the components in a mixture of drugs in order to prevent selection for resistance in a more general context of biocide resistance.

\section{ACKNOWLEDGMENTS}

A. Mikaberidze and S. Bonhoeffer gratefully acknowledge support by the European Research Council advanced grant PBDR 268540. We thank M. Milgroom and M. Shaw for helpful comments concerning fitness costs of fungicide resistance and two anonymous reviewers for improving the manuscript.

\section{LITERATURE CITED}

1. Abbott, W. 1925. A method of computing the effectiveness of an insecticide. J. Econ. Entomol. 18:265.

2. Anderson, R. M., and May, R. M. 1986. The invasion, persistence and 
spread of infectious diseases within animal and plant communities. Phil. Trans. Roy. Soc. London. Series B, Biol. Sci. 314:533-570.

3. Andersson, D. I., and Hughes, D. 2010. Antibiotic resistance and its cost: Is it possible to reverse resistance? Nat. Rev. Microbiol. 8:260-271.

4. Austin, D. J., and Anderson, R. M. 1999. Studies of antibiotic resistance within the patient, hospitals and the community using simple mathematical models. Phil. Trans. Roy. Soc. London. Series B, Biol. Sci. 354:721-738.

5. Beckie, H. J., and Reboud, X. 2009. Selecting for weed resistance: Herbicide rotation and mixture. Weed Technol. 23:363-370.

6. Bell, G., and Gonzalez, A. 2011. Adaptation and evolutionary rescue in metapopulations experiencing environmental deterioration. Science 332: 1327-1330.

7. Berenbaum, M. 1989. What is synergy? Pharmacol. Rev. 41:93.

8. Billard, A., Fillinger, S., Leroux, P., Lachaise, H., Beffa, R., and Debieu, D. 2012. Strong resistance to the fungicide fenhexamid entails a fitness cost in Botrytis cinerea, as shown by comparisons of isogenic strains. Pest Manag. Sci. 68:684-691.

9. Bliss, C. I. 1939. The toxicity of poisons applied jointly. Ann. Appl. Biol. 26:585-615.

10. Bonten, M. J. M., Austin, D. J., and Lipsitch, M. 2001. Understanding the spread of antibiotic resistant pathogens in hospitals: Mathematical models as tools for control. Healthcare Epidemiol. 33:1739-1746.

11. Chait, R., Craney, A., and Kishony, R. 2007. Antibiotic interactions that select against resistance. Nature 446:668-671.

12. Cools, H. J. 2008. Are azole fungicides losing ground against Septoria wheat disease? Resistance mechanisms in Mycosphaerella graminicola. Pest Manag. Sci. 64:681-684.

13. Corio-Costet, M.-F., Dufour, M.-C., Cigna, J., Abadie, P., and Chen, W.-J. 2010. Diversity and fitness of Plasmopara viticola isolates resistant to QoI fungicides. Eur. J. Plant Pathol. 129:315-329.

14. Fernández-Ortuño, D., Torés, J. A., Vicente, A. D., and Pérez-Garca, A. 2008. Mechanisms of resistance to QoI fungicides in phytopathogenic fungi. Intl. Microbiol. 11:1-9.

15. Gisi, U. 1996. Synergistic interaction of fungicides in mixtures. Phytopathology 86:1273.

16. Gisi, U., Sierotzki, H., Cook, A., and McCaffery, A. 2002. Mechanisms influencing the evolution of resistance to Qo inhibitor fungicides. Pest Manag. Sci. 58:859-867.

17. Greco, W., and Bravo, G. 1995. The search for synergy: A critical review from a response surface perspective. Pharmacol. Rev. 47:331.

18. Gubbins, S., and Gilligan, C. A. 1999. Invasion thresholds for fungicide resistance: Deterministic and stochastic analyses. Proc. Roy. Soc. Lond. B 266:2539-2549.

19. Hall, R. J., Gubbins, S., and Gilligan, C. A. 2004. Invasion of drug and pesticide resistance is determined by a trade-off between treatment efficacy and relative fitness. Bull. Math. Biol. 66:825-840.

20. Hall, R. J., Gubbins, S., and Gilligan, C. A. 2007. Evaluating the performance of chemical control in the presence of resistant pathogens. Bull. Math. Biol. 69:525-537.

21. Hobbelen, P. H. F., Paveley, N. D., Oliver, R. P., and van den Bosch, F. 2013. The usefulness of fungicide mixtures and alternation for delaying the selection for resistance in populations of Mycosphaerella graminicola on winter wheat: A modeling analysis. Phytopathology 103:690-707.

22. Hobbelen, P. H. F., Paveley, N. D., and van den Bosch, F. 2011. Delaying selection for fungicide insensitivity by mixing fungicides at a low and high risk of resistance development: A modeling analysis. Phytopathology 101:1224-1233.

23. Hoffmann, A. A., and Sgrò, C. M. 2011. Climate change and evolutionary adaptation. Nature 470:479-485.

24. Holmes, G., and Eckert, J. 1995. Relative fitness of imazalil-resistant and -sensitive biotypes of Penicillium digitatum. Plant Dis. 79:1068-1073.

25. Iacomi-Vasilescu, B., Bataille-Simoneau, N., Campion, C., Dongo, A., Laurent, E., Serandat, I., Hamon, B., and Simoneau, P. 2008. Effect of null mutations in the AbNIK1 gene on saprophytic and parasitic fitness of Alternaria brassicicola isolates highly resistant to dicarboximide fungicides. Plant Pathol. 57:937-947.

26. Josepovits, G., and Dobrovolszky, A. 1985. A novel mathematical approach to the prevention of fungicide resistance. Pest. Sci. 16:17-22.

27. Kable, P., and Jeffery, H. 1980. Selection for tolerance in organisms exposed to sprays of biocide mixtures: A theoretical model. Phytopathology 79:8-12.

28. Kadish, D., and Cohen, Y. 1989. Population dynamics of metalaxylsensitive and metalaxyl-resistant isolates of Phytophthora infestans in untreated crops of potato. Plant Pathol. 38:271-276.

29. Kadish, D., and Cohen, Y. 1992. Overseasoning of metalaxyl-sensitive and metalaxyl-resistant isolates of Phytophthora infestans in potato tubers weeks after inoculation. Phytopathology 82:887-889.
30. Karaoglanidis, G., Thanassoulopoulos, C., and Ioannidis, P. 2001. Fitness of Cercospora beticola field isolates resistant and sensitive to demethylation inhibitor fungicides. Eur. J. Plant Pathol. 107:337-347.

31. Kim, Y. K., and Xiao, C. L. 2011. Stability and fitness of pyraclostrobinand boscalid-resistant phenotypes in field isolates of Botrytis cinerea from apple. Phytopathology 101:1385-1391.

32. Leroux, P., Albertini, C., Gautier, A., Gredt, M., and Walker, A.-S. 2007. Mutations in the CYP51 gene correlated with changes in sensitivity to sterol $14 \alpha$-demethylation inhibitors in field isolates of Mycosphaerella graminicola. Pest Manag. Sci. 63:688-698.

33. Levin, B. R. 2001. Minimizing potential resistance: A population dynamics view. Clin. Infect. Dis. 33:161-169.

34. Levy, Y., Benderly, M., Cohen, Y., Gisi, U., and Bassand, D. 1986. Joint action of fungicides in mixtures: comparison of two methods for synergy calculation. EPPO Bull. 16:651-657.

35. Lockley, D., and Clark, W. 2005. Fungicide dose-response trials in wheat: The basis for choosing "appropriate dose". Home-Grown Cereals Authority, UK, Project Report 373.

36. Milgroom, M. G., Levin, S. A., and Fry, W. E. 1989. Population genetics theory and fungicide resistance. Pages 340-367 in: Plant Disease Epidemiology. 2. Genetics, Resistance and Management. K. J. Leonard and W. E. Fry, eds. McGraw Hill, New York.

37. Mullins, J. G. L., Parker, J. E., Cools, H. J., Togawa, R. C., Lucas, J. A., Fraaije, B. A., Kelly, D. E., and Kelly, S. L. 2011. Molecular modelling of the emergence of azole resistance in Mycosphaerella graminicola. PloS One 6:e20973.

38. Orton, E. S., Deller, S., and Brown, J. K. M. 2011. Pathogen profile update Mycosphaerella graminicola: From genomics to disease control. Mol. Plant Pathol. 12:413-424.

39. Ozcaglar, C., Shabbeer, A., Vandenberg, S. L., Yener, B., and Bennett, K. P. 2012. Epidemiological models of Mycobacterium tuberculosis complex infections. Math. Biosci. 236:77-96.

40. Palmer, C., and Skinner, W. 2002. Mycosphaerella graminicola: Latent infection, crop devastation and genomics. Mol. Plant Pathol. 3:63-70.

41. Parnell, S., Gilligan, C. A., and van den Bosch, F. 2005. Small-scale fungicide spray heterogeneity and the coexistence of resistant and sensitive pathogen strains. Phytopathology 95:632-639.

42. Paveley, N., Hims, M., and Stevens, D. 1998. Appropriate fungicide doses for winter wheat and matching crop management to growth and yield potential. Home-Grown Cereals Authority, UK, Project Report 166.

43. Peever, T., and Milgroom, M. 1995. Fungicide resistance-Lessons for herbicide resistance management? Weed Technol. 9:840.

44. Peever, T. L., and Milgroom, M. G. 1994. Lack of correlation between fitness and resistance to sterol biosynthesis-inhibiting fungicides in Pyrenophora teres. Phytopathology 84:515-519.

45. Pfender, W. 2006. Interaction of fungicide physical modes of action and plant phenology in control of stem rust of perennial ryegrass grown for seed. Plant Dis. 90:1225.

46. Roush, R. 2006. Designing resistance management programs: How can you choose? Pest. Sci. 26:15-16.

47. Shaw, M. 1989. Independent action of fungicides and its consequences for strategies to retard the evolution of fungicide resistance. Crop Prot. 8:405411.

48. Shaw, M. 1993. Theoretical analysis of the effect of interacting activities on the rate of selection for combined resistance to fungicide mixtures. Crop Prot. 12:120-126.

49. Shaw, M. W. 1989. A model of the evolution of polygenically controlled fungicide resistance. Plant Pathol. 38:44-55

50. Shaw, M. W. 2006. Is there such a thing as a fungicide resistance strategy? A modeller's perspective. Aspects Appl. Biol. 78:37-43.

51. Skylakakis, G. 1981. Effects of alternating and mixing pesticides on the buildup of fungal resistance. Phytopathology 71:1119.

52. Suzuki, F., Yamaguchi, J., and Koba, A. 2010. Changes in fungicide resistance frequency and population structure of Pyricularia oryzae after discontinuance of MBI-D fungicides. Plant Dis. 94:329-334.

53. Torriani, S. F., Brunner, P. C., McDonald, B. A., and Sierotzki, H. 2009. QoI resistance emerged independently at least 4 times in European populations of Mycosphaerella graminicola. Pest Manag. Sci. 65:155-162.

54. van den Bosch, F., and Gilligan, C. A. 2008. Models of fungicide resistance dynamics. Annu. Rev. Phytopathol. 46:123-147.

55. Webber, J. 1988. Effect of MBC fungicide tolerance on the fitness of Ophiostoma ulmi. Plant Pathol. 37:217-224.

56. Wong, F., and Wilcox, W. 2001. Comparative physical modes of action of azoxystrobin, mancozeb, and metalaxyl against Plasmopara viticola (grapevine downy mildew). Plant Dis. 85:649-656.

57. Zhan, J., Stefanato, F., and McDonald, B. 2006. Selection for increased cyproconazole tolerance in Mycosphaerella graminicola through local adaptation and in response to host. Mol. Plant Pathol. 7:259-268. 